\title{
On the effects of surrogacy of energy dissipation in determining the intermittency exponent in fully developed turbulence
}

\author{
J. Cleve $\mathrm{e}^{1,2,3}$, M. Greiner ${ }^{4}$, K. R. Sreenivasan ${ }^{1}$ \\ ${ }^{1}$ Institute for Physical Science and Technology, University of Maryland, College Park, MD 20742, USA \\ ${ }^{2}$ Max-Planck-Institut für Physik komplexer Systeme, Nöthnitzer Str. 38, D-01187 Dresden, Germany \\ ${ }^{3}$ Institut für Theoretische Physik, Technische Universität Dresden, D-01062 Dresden, Germany and \\ ${ }^{4}$ Corporate Technology, Information \& Communications, Siemens AG, D-81730 München, Germany
}

\begin{abstract}
The two-point correlation function of the energy dissipation, obtained from a one-point time record of an atmospheric boundary layer, reveals a rigorous power-law scaling with intermittency exponent $\mu \approx 0.20$ over almost the entire inertial range of scales. However, for the related integral moment, the power-law scaling is restricted to the upper part of the inertial range only. This observation is explained in terms of the operational surrogacy of the construction of energy dissipation, which influences the behaviour of the correlation function for small separation distances.
\end{abstract}

PACS: 47.27.Jv High-Reynolds-number turbulence;

05.40.-a Fluctuation phenomena, random processes, noise, and Brownian motion; 02.50.Sk Multivariate analysis

Time records of turbulent velocity at a single point in space, obtained using a hot-wire or a laser Doppler anemometer, are usually interpreted, via Taylor's frozen flow hypothesis, as one-dimensional spatial cuts through the flow. Velocity structure functions can be obtained readily from such observables. In addition to the velocity, other quantities of interest include enstrophy and energy dissipation. These quantities cannot be constructed in full from the measured one-point velocity time series (of one or two components of velocity) and so are replaced for further analysis by the so-called surrogate fields. These surrogate fields usually take the form of a single component of a many-component field. In this note we concentrate on the surrogacy issue of energy dissipation and discuss its impact on the extraction of the intermittency exponent.

To illustrate the issue, we choose turbulence measurements in an atmospheric boundary layer, made under nominally steady and nearly neutral conditions, in which a hot-wire probe mounted on top of a tower recorded time-series of both streamwise and vertical velocity components; for details of the experimental setup, see Ref. 1]. The frozen flow hypothesis has been applied to convert the time series into spatial cuts. Upon using the method of Ref. [2], the Reynolds number $R_{\lambda}=\sqrt{\left\langle u^{2}\right\rangle} \lambda / \nu$, based on the Taylor microscale $\lambda=\sqrt{\left\langle u^{2}\right\rangle /\left\langle(\partial u / \partial x)^{2}\right\rangle}$, was determined to be 9000 . The angular brackets denote a temporal average throughout the paper. The estimated ratio between integral length $L$, defined through the integral of the two-point correlation of the component velocity fluctuation in the streamwise direction, and the dissipation scale $\eta=\left(\nu^{3} /\langle\varepsilon\rangle\right)^{1 / 4}$, is $5 \times 10^{4}$. In units of $L$ the record length of the time series is $L_{\text {record }} / L=1000$. The inertial range of scales is determined by examining the scaling of the third-order structure function; within the inertial range so determined, the power spectra show a well-defined slope close to $-5 / 3$. Because of instrument and cable noise, the spectral density has some amount of noise contamination towards the smallest scales. In order to ensure a proper construction of the derivatives $\partial v_{i} / \partial x=\left(v_{i}(x+\Delta x)-v_{i}(x)\right) / \Delta x$, the noise part has been removed from the velocity signal by using a Wiener filter.

The true energy dissipation rate

$$
\varepsilon(\vec{x})=\frac{\nu}{2} \sum_{i, j}\left(\partial_{i} v_{j}+\partial_{j} v_{i}\right)^{2}
$$

where the indices $i$ and $j$ represent the coordinate axes, cannot be constructed from the recorded time series. This is so because only the longitudinal and transverse components $v_{x}$ and $v_{y}$ of the velocity, along the streamwise direction $x$ and the normal direction $y$ respectively, are accessible. Hence, the true energy dissipation is replaced by a surrogate proposal. One possibility is

$$
\varepsilon_{\text {surr1 }}(x)=15 \nu\left(\partial_{x} v_{x}(x)\right)^{2},
$$

which, upon assuming isotropy, equals true dissipation on the average.

For the extraction of the intermittency exponent, the two-point correlation function based on the energy dissipation field has been proposed earlier [3]. Figure [1 shows the normalized two-point correlator $\left\langle\varepsilon_{\text {surr } 1}(x+\right.$ d) $\left.\varepsilon_{\text {surr } 1}(x)\right\rangle /\left\langle\varepsilon_{\text {surr1 }}(x)\right\rangle^{2}$. It reveals a good scaling $\sim d^{-\mu}$ with constant intermittency exponent, $\mu=0.20$, over the extended scaling range $15 \eta \leq d \leq 0.3 L$, covering most of the inertial range. This result is in full agreement with older findings on turbulent jet and atmospheric boundary layer flows [4, 5], which have also observed a clear 


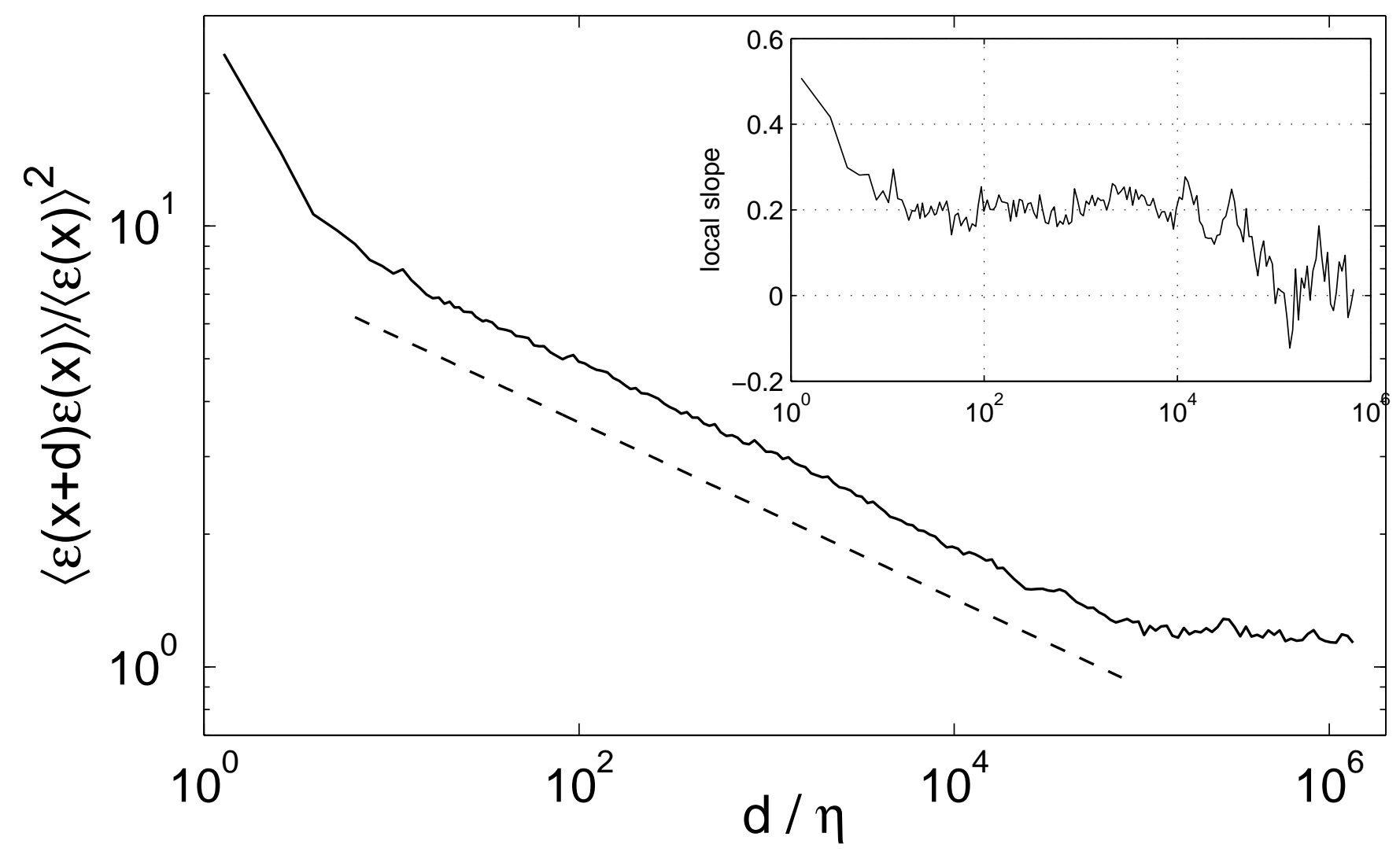

FIG. 1: Normalized two-point correlation function of the surrogate energy dissipation field (2) obtained via the streamwise velocity component in the atmosphere. The dashed line has a logarithmic slope $\mu=0.2$ and its extent indicates the scaling range. Inset: logarithmic local slope of the two-point correlation function.

and extended scaling range behaviour for the two-point correlator with about the same value for the intermittency exponent.

The two-point correlator of the surrogate energy dissipation is only one tool for the extraction of the intermittency exponent. Another approach that is natural from the perspective of the multifractal picture of turbulence [6, 7, 8$]$ employs the second-order moment $\left\langle\varepsilon_{l}^{2}\right\rangle$ of the coarse-grained amplitude $\varepsilon_{l}=l^{-1} \int_{l} \varepsilon_{\text {surr }}(x) d x$. Figure 2 shows $\left\langle\varepsilon_{l}^{2}\right\rangle$ as a function of the averaging scale $l$. The logarithmic local slope $-d \ln \left\langle\varepsilon_{l}^{2}\right\rangle / d \ln l$ reveals a scale-independent scaling exponent, which coincides roughly with the previously found value $\mu=0.20$ for the intermittency exponent. However, a peculiarity remains: the logarithmic local slope in Fig. 2] is constant (or nearly so) only in the upper part of the inertial range, and not over the full range, as found for the two-point correlator. One purpose of this paper is to understand this puzzle.

To proceed, we note that the second-order moment and the two-point correlator are closely related (see [3]) via

$$
\left\langle\varepsilon_{l}^{2}\right\rangle=\frac{1}{l^{2}} \int_{l} d x_{1} \int_{l} d x_{2}\left\langle\varepsilon_{\text {surr }}\left(x_{1}\right) \varepsilon_{\text {surr }}\left(x_{2}\right)\right\rangle .
$$

The left-hand-side represents a box integral over the two-point correlator and may thus be called an integral moment. To see when the integral moment shows the same scaling exponent as the two-point correlator, and in what range of scales, we assume the simplified functional form

$$
\left\langle\varepsilon_{\mathrm{surr}}(x+d) \varepsilon_{\mathrm{surr}}(x)\right\rangle= \begin{cases}c & \left(d<\eta^{\prime}\right) \\ a\left(\eta^{\prime} / d\right)^{\mu} & \left(\eta^{\prime} \leq d \leq L^{\prime}\right) \\ 1 & \left(d \geq L^{\prime}\right)\end{cases}
$$

with $\left\langle\varepsilon_{\text {surr }}\right\rangle=1$, where $a\left(\eta^{\prime} / L^{\prime}\right)^{\mu}=1$ guarantees continuity at the decorrelation length $d=L^{\prime}$; the parameter $c$ is left free for later purposes and $\eta^{\prime}$ and $L^{\prime}$ are representative small and large length scale, respectively. Upon inserting (4) 


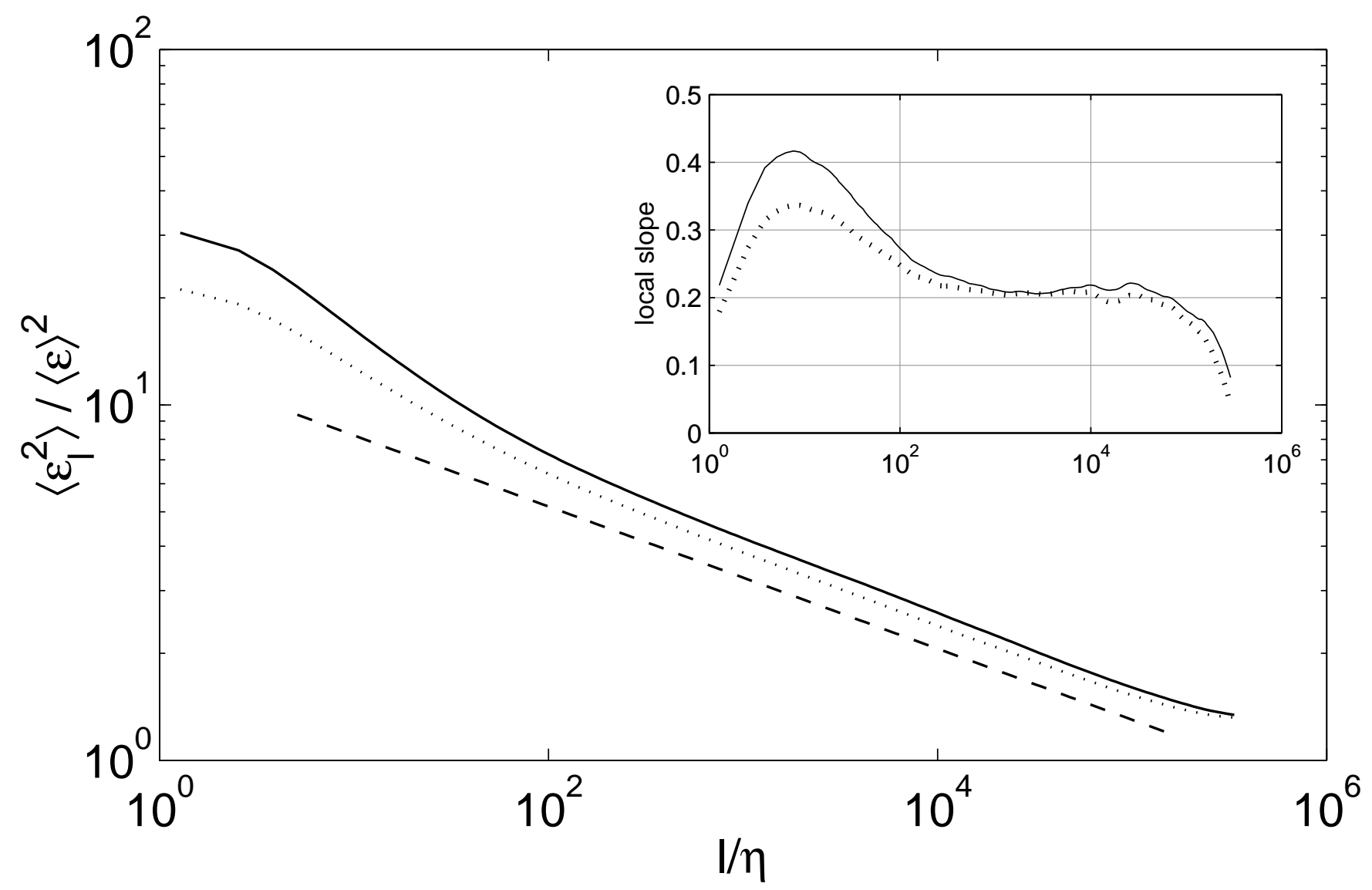

FIG. 2: Second-order integral moment (3) based on the surrogate energy dissipation fields (2) (full line), and (7) (dotted line). The dashed straight line has a logarithmic slope $\mu=0.2$. Inset shows the logarithmic local slope.

into (3) we arrive at

$$
\left\langle\varepsilon_{l}^{2}\right\rangle=\frac{2 a}{(1-\mu)(2-\mu)}\left(\frac{\eta^{\prime}}{l}\right)^{\mu}+2\left(c-\frac{a}{1-\mu}\right)\left(\frac{\eta^{\prime}}{l}\right)+\left(\frac{a}{1-\mu / 2}-c\right)\left(\frac{\eta^{\prime}}{l}\right)^{2},
$$

valid for $\eta^{\prime} \leq l \leq L^{\prime}$. The first term is the targeted scaling term. The last two terms represent corrections to rigorous scaling. For increasing $l \gg \eta^{\prime}$ they fall off faster than the scaling term. If we were to fine-tune the leading-order correction to zero, then $c=a /(1-\mu)$. Given that $\mu=0.20$, the correction to the leading order term also becomes very small. A closer look at Fig. 11 reveals that a constant $c=a /(1-\mu)$ is too small to approximate the small-scale behaviour of the two-point correlation. Consequently, the correction terms are pronounced for small $l$ and extend far into the inertial range before becoming negligible. This explains qualitatively the observed scale-dependence of the second-order integral moment: only in the upper part of the inertial range does the scaling term with exponent $\mu$ dominate, whereas for the lower part strong deviations set in, due to the behaviour of the two-point correlation function for very small scales.

Figure 1 1 reveals another apparent puzzle: as the two-point distance approaches $\eta$ the two-point correlation increases stronger than is suggested by the extrapolation of scaling behaviour. This is against intuition, since the onset of dissipation is expected to smooth out the fine structure, instead of building it up. We now offer a tentative explanation, which reflects the delicate issue of the surrogacy of the energy dissipation field. The two-point correlator shown in Fig. 1 is based on the surrogate energy dissipation field (2) constructed from the longitudinal velocity component. Other constructions, such as

$$
\begin{aligned}
& \varepsilon_{\mathrm{surr} 2}(x)=\frac{15}{2} \nu\left(\partial_{x} v_{y}(x)\right)^{2} \\
& \varepsilon_{\mathrm{surr} 3}(x)=\frac{15}{4} \nu\left[2\left(\partial_{x} v_{x}(x)\right)^{2}+\left(\partial_{x} v_{y}(x)\right)^{2}\right]
\end{aligned}
$$




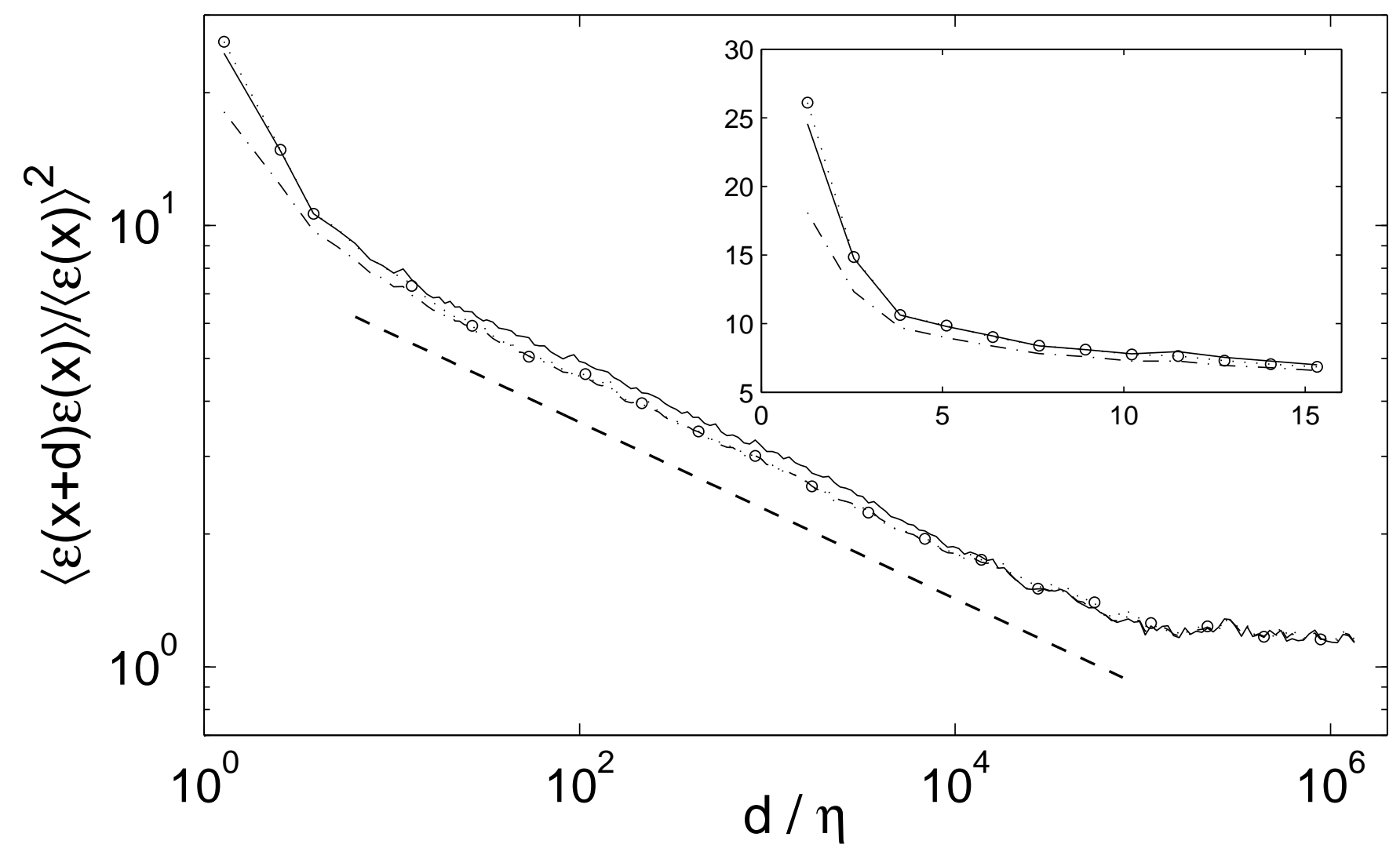

FIG. 3: Normalized two-point correlation function of the surrogate energy dissipation fields (2) (full line), (6) (dotted line with circles), and (7) (dot-dashed line). The dashed straight line has a logarithmic slope $\mu=0.2$. Inset magnifies the behaviour for short separation distances.

are also possible. The former is based on the transverse velocity component alone, whereas the latter combines longitudinal and transverse components. On average, both constructions are equal in their mean value to the true energy dissipation field (1), assuming isotropy.

Figure 3 compares the two-point correlator obtained from the surrogate quantities (2), (6) and (7). All three variants reveal a rigorous power-law scaling behaviour within the extended inertial range $15 \eta \leq d \leq 0.3 L$ and the corresponding scaling exponents are within $\mu=0.20 \pm 0.01$, showing little differences. Only for small two-point distances $d \rightarrow \eta$ the two-point correlators begin to differ. Whereas the variants based on (2) and (6) practically remain identical, the two-point correlations based on (7) are weaker for $d \leq 10 \eta$; see inset of Fig. 3] In view of the simplified description (4) this implies that the constant $c$ is smaller for the two-point correlator based on (7) than those based on (2) and (6). As a consequence the leading order corrections in the expression (5) for the integral moment also become smaller, so that the scaling term should begin to dominate even at smaller length scales $l$. Figure 2 confirms this view: the local slope of the integral moment based on the surrogate field (7) becomes constant at smaller scales than for (2); the upper limit on the scaling range is the same for the two cases.

When compared to the true energy dissipation (1), the surrogate (17) appears to be closer to (1) than the other two variants (2) and (6). We might model the amplitude of the surrogate field in terms of the amplitude of the true field by the relationship

$$
\varepsilon_{\mathrm{surr}}(x)=\varepsilon(x)(1+f(x))
$$

where $f(x)$ behaves as a noise with mean zero. From the defining equations (2), (6) and (7) we get $\varepsilon_{\text {surr3 }}=\left(\varepsilon_{\text {surr1 }}+\right.$ $\left.\varepsilon_{\text {surr } 2}\right) / 2=\varepsilon\left[1+\left(f_{1}+f_{2}\right) / 2\right]$, so that in comparison with $f_{1}$ and $f_{2}$ the noise fluctuation $f_{3}=\left(f_{1}+f_{2}\right) / 2$ is reduced. Hence, this allows us to speculate that if we add more terms from the full list of (11), the extra-strong two-point correlations at small separation distances $d \leq 15 \eta$ reduce further, perhaps even vanish once the surrogate field has converged to the true field. A quick numerical investigation, using a shear turbulence code 9$]$ for $R_{\lambda}=99$, reveals that the two-point correlation functions of the surrogate quantities are identical to that of true energy dissipation, except for very small distances, where the surrogate field possesses extra-strong correlations. While a more detailed 


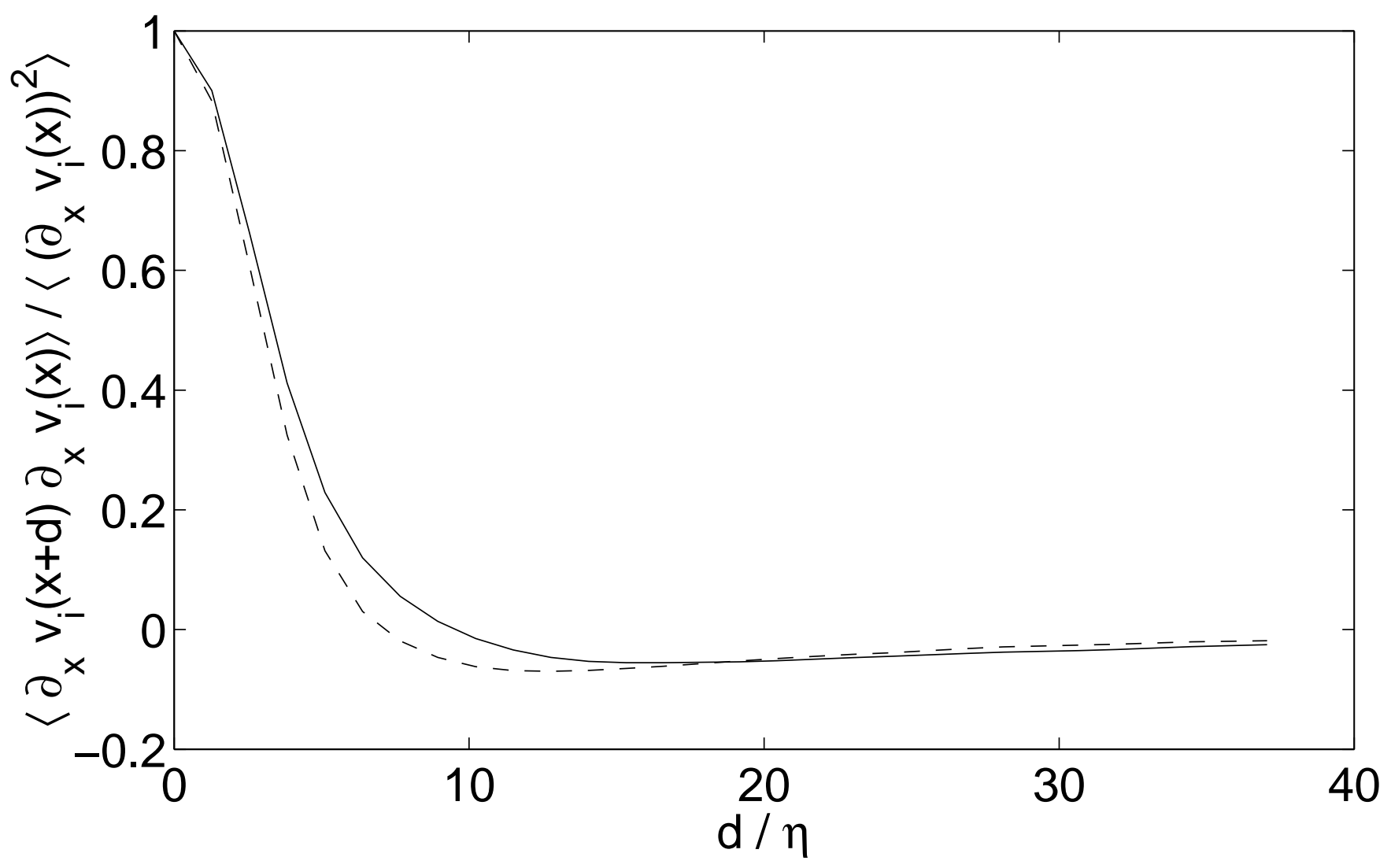

FIG. 4: Normalized two-point correlations $\left\langle\left(\partial_{x} v_{i}(x+d)\right)\left(\partial_{x} v_{i}(x)\right)\right\rangle /\left\langle\left(\partial_{x} v_{i}(x)\right)^{2}\right\rangle$ of the longitudinal (full line, $\left.v_{i}=v_{x}\right)$ and transverse (dashed, $\left.v_{i}=v_{y}\right)$ velocity gradients.

investigation is called for, this finding indicates the importance of the subtle surrogacy issue when interpreting data. It appears clear that the surrogacy of the energy dissipation field restricts the rigorous scaling of the second-order integral moment to the upper part of the inertial range; fortunately, this leaves the rigorous scaling of the two-point correlation function untouched over that part of the inertial range.

Considering the behaviour of the surrogate correlators for small separation distances in terms of the modeling relation (8), the noise field amplitudes cannot be expected to be uncorrelated, i.e. $\left\langle f\left(x_{1}\right) f\left(x_{2}\right)\right\rangle \neq\left\langle f^{2}\right\rangle \delta\left(x_{1}-x_{2}\right)$, but should show correlations up to some separation distance. Empirically, this seems to occur within the range $\left|x_{1}-x_{2}\right| \approx 15 \eta$. For shorter distances, the extra-strong correlation sets in for the two-point correlators based on the surrogate fields. A quantity related to the noise correlations is the two-point correlation of the velocity gradient field. Figure 4 shows $\left\langle\left(\partial_{x} v_{i}(x+d)\right)\left(\partial_{x} v_{i}(x)\right)\right\rangle$ for the measured longitudinal $\left(v_{i}=v_{x}\right)$ and transverse $\left(v_{i}=v_{y}\right)$ velocity components. All two-point correlations show correlations up to $d \approx 30 \eta$ and become zero for larger distances.

The main message of this simple view on the surrogacy issue of the energy dissipation field is two-fold: (i) Twopoint correlation functions of the surrogate energy dissipation field reveal rigorous scaling that is identical to the two-point correlation function of the true energy dissipation for two-point distances larger than about fifteen times the dissipation scale. (ii) The surrogacy of the energy dissipation modifies the two-point correlations for distances below $d \approx 15 \eta$ and is responsible for restricting the power-law scaling behaviour of the integral moment to the upper part of the inertial range. A direct consequence of statements (i) and (ii) is that for the extraction of the intermittency exponent it is advantageous to use the two-point correlator. In view of the extended scaling range $15 \eta \leq d \leq 0.3 L$ of the two-point correlator of the energy dissipation, it appears that the intuitive, but phenomenological, picture of the scale-invariant energy cascade contains more truth than generally anticipated. Without further processing such as Extended Self Similarity [10] or $S O(3)$ decomposition [11, 12, 13], conventional velocity structure functions in shear flows do not show a rigorous power-law scaling behaviour. This assigns fields such as the energy dissipation a more fundamental role than that of the velocity increments. As this inference is based on data analysis alone, it does not offer any deep theoretical explanation but requires one. 


\section{Acknowledgments}

The authors acknowledge fruitful discussions with Jürgen Schmiegel, Thomas Dziekan, Hans Eggers, Jörg Schumacher, Jahanshah Davoudi, Jean-François Pinton and Joachim Peinke. J.C. acknowledges support from DAAD.

[1] B. Dhruva, An Experimental Study of High Reynolds Number Turbulence in the Atmosphere, PhD thesis, Yale University (2000); see also K. R. Sreenivasan and B. Dhruva, Prog. Theor. Phys. Suppl. 130, 103 (1998).

[2] D. Aronson and L. Löfdahl Phys. Fluids A 6, 1433 (1993).

[3] A.S. Monin and A.M. Yaglom, Statistical Fluid Mechanics, Vol. 2 (MIT Press, Cambridge, 1971).

[4] R. A. Antonia, B. R. Satyaprakash and A. K. M. F. Hussain J. Fluid Mech. 119, 55 (1982).

[5] K. R. Sreenivasan and P. Kailasnath Phys. Fluids A 5, 512 (1993).

[6] B. Mandelbrot J. Fluid Mech. 62, 331 (1974).

[7] C. Meneveau and K. R. Sreenivasan J. Fluid Mech. 224, 429 (1991).

[8] U. Frisch Turbulence (Cambridge University Press, Cambridge, 1995).

[9] J. Schumacher J. Fluid Mech. 441, 109 (2001).

[10] R. Benzi, S. Ciliberto, R. Tripiccione, C. Baudet, F. Massaioloi and S. Succi Phys. Rev. E 48, 29 (1993).

[11] V. S. L'vov, E. Podivilov and I. Procaccia Phys. Rev. Lett. 79, 2050 (1997).

[12] I. Arad, B. Dhruva, S. Kurien, V. S. L'vov, I. Procaccia and K. R. Sreenivasan Phys. Rev. Lett. 81, 5330 (1998).

[13] S. Kurien and K. R. Sreenivasan New Trends in Turbulence, Les Houches Summer School Proceedings 2000 (Springer-EDP, Paris, 2001) edited by M.Lesieur, p. 51-111 